\title{
Substanzen aus der philosophischen Apotheke
}

\section{Jann P. Schwarzenbach}

Dr. med., Facharzt für Allgemeinmedizin, Mitglied FMH

Unter Substanzen verstehen wir Ärzte und Ärztinnen normalerweise chemisch-pharmakologische Stoffe, von denen wir dann wenigstens hoffen, dass sie die angepriesenen günstigen Effekte zum Vorteil unserer Patienten auch ausüben werden. Man kann den Begriff der Substanz aber auch ganz anders auffassen. Nicht als etwas Dynamisches und Wirkkräftiges, sondern im Gegenteil als eine den Dingen innewohnende Trägheit und Unverrückbarkeit: ein Fixpunkt und Ruhepol inmitten variabler Eigenschaften und situativer Begebenheiten. So bleibt der hauseigene Vierbeiner immer "unser Hund», ob er nun als wedelnder Wollknäuel oder als knurrender Postbotenschreck erscheinen mag. Auch was unser Selbst anbetrifft, setzen wir natürlicherweise etwas Konstantes voraus: ein Ich als Träger der charakterlichen Eigenschaften und als Protagonist des eigenen Lebenslaufs. So bewahren wir beispielsweise in unserem Inneren - allen seelischkörperlichen Veränderungen zum Trotz - ein erstaunlich zeitresistentes Selbstverständnis: vom erinnerten Studenten-Ich bis hin zum aktuellen Berufs- oder Pensionärs-Ich. Diese Gewissheit einer dauerhaften persönlichen Identität veredelt unsere tägliche Selbsterfahrung und kann gleichzeitig hilfreich sein, unsere Patienten in ihrer individuellen Besonderheit zu verstehen und zu respektieren.

\section{Metaphysika und Empirika}

Die Natur der Substanz beziehungsweise die Frage, ob es ein unveränderliches "Etwas» gibt, das einem Gegenstand oder einer Person abgesehen von allen zeitlich und örtlich wandelbaren Eigenschaften innewohnt, hat die Philosophen seit jeher beschäftigt.

Wir setzen etwas Konstantes voraus: ein Ich als Träger der charakterlichen Eigenschaften und als Protagonist des eigenen Lebenslaufs.

So war Plato von der Existenz individueller Substanzen als zeitlose Essenzen aller Dinge überzeugt, hat diese aber gleichzeitig in einer Art von «Cloud» ausgelagert und damit als metaphysische, rein ideelle Einheiten der empirischen Erfahrbarkeit entzogen. Die Substanz eines Menschen war in diesem Sinne identisch mit seiner angeblich seit jeher präexistierenden, unsterblichen Seele. Aristoteles, dessen Philosophie dem Allgemeinempfinden um einiges näher liegt, fand Substanzielles im konkret vorhandenen Einzelnen und in den diesem letzteren notwendigerweise zugehörigen Attributen. Bei einem Exemplar des Homo sapiens wären das beispielsweise der angeborene Charakter und die exklusive Fähigkeit, rational zu denken. Die an die Substanz gekoppelten Akzidentien hingegen bestimmen nach Aristoteles die variable, empirisch erfahrbare Form, in der die einzelnen Dinge, und so auch der Mensch, von Fall zu Fall erscheinen. Bei Vertretern unserer Spezies handelt es sich dabei etwa um Parameter wie Körpergewicht, Alter, Besitz, Beruf und sozialen Status. In den auf die Antike folgenden, von religiösem Denken bestimmten Jahrhunderten gab es das Substanzielle nur noch von Gottes Gnaden, bis dann die moderneren Philosophen von Descartes bis Hegel es in unserem Bewusstsein, beziehungsweise unserem Selbstbewusstsein, aufzufinden glaubten. Für die damalige Zeit unerhört skeptische Betrachtungen zum Thema finden wir in den Schriften von David Hume (1711-1776), auf den ich im nächsten Abschnitt ein wenig näher eingehen werde. Er spricht den Substanzen jede wahre Realität ab und reduziert sie auf ein rein empirisch-psychologisches Phänomen, das dann auftritt, wenn wir gewisse Dinge über längere Zeit beobachten. Man könnte jetzt natürlich die Diskussion über die tatsächliche oder eben nur eingebildete Existenz von Substanzhaftem als eine Schrulle von Philosophen betrachten, die locker die nötige Zeit aufbringen, sich mit solchen akademischen Spitzfindigkeiten auseinanderzusetzen. Ginge es in diesem Zusammenhang nur um die wesenhafte Beständigkeit von Alltagsobjekten oder einfachen Lebewesen, wäre diese Kritik berechtigt. Wenn wir hingegen vom Menschen und hier in besonderem Masse auch von unseren Patienten sprechen, so zeigt die Thematik grosse praktische Relevanz: Es geht hier nämlich um die Frage, ob wir über 
eine substanzielle innere Identität verfügen, ob es ein stabiles Ich gibt, das uns ein Leben lang begleitet. Ein Ich, das auch in Krankheit und Alter, sowie vor und

Hat die Frau des Alkoholikers doch recht, wenn sie uns klagt, nicht mehr denselben Mann zu Hause zu haben, den sie einst geheiratet hat?

nach einer ärztlichen Behandlung, grundsätzlich dasselbe bleibt. Auf diese für alle medizinisch Tätigen wichtige Thematik werde ich im letzten Abschnitt noch zurückkommen.

\section{Nur Bündel von Wahrnehmungen}

Zunächst jetzt aber ein paar Zeilen zu David Humes kompromisslosem Empirismus, der gerade in der heutigen, wissenschaftlich geprägten Zeit uns stets zu denken geben sollte: Alle Erkenntnis verdanken wir nach der Meinung dieses Philosophen einzig und allein unserer Wahrnehmung. So komme denn auch reale Existenz nur unseren «impressions» und «ideas» zu, das heisst den primären Sinneseindrücken und deren im Gedächtnis haften gebliebenen Abbildern. Wenn in unserem Bewusstsein verschiedene einfache Eindrücke wiederholt nebenoder kurz nacheinander entstehen, so seien wir durch unsere Einbildungskraft befähigt, diese Ansammlung von Sinnesdaten als Gegenstand wahrzunehmen und als zusammengesetzte Idee in unserem Gedächtnis abzuspeichern. Keinesfalls aber könne man, ausgehend von solchen mentalen Konstrukten, auf das Vorhandensein selbständiger Objekte in der Aussenwelt schliessen. Die dem Menschen eigene, spontane Gewissheit einer bewusstseinsexternen Realität sei nur Folge der besonderen Intensität einzelner Ideen und Eindrücke und müsse letztlich als ein blosser Glaube angesehen werden. Dies steht natürlich unserem Alltagsverständnis diametral entgegen, ist aber eine logische Folge der konsequenten Empirie. Analog dazu spricht Hume den Substanzen jegliche reale Existenz $\mathrm{ab}$, sie sind für ihn nur besonders lange dauernde Ansammlungen von Ideen, also von den im Gedächtnis eingelagerten Sinneseindrücken. Somit wäre denn auch die Diskussion hinsichtlich der Existenz eines eventuellen zeitresistenten menschlichen Ichs hinfällig und unsere persönliche Identität nicht mehr als ein durch unsere Vorstellungskraft immer wieder frisch geschnürtes Bündel wechselhafter «impressions» und «ideas».

\section{Garanten der schutzwürdigen Identität}

Ist die Krankheit nur ein aristotelisches Akzidens einer substanziell gleichgebliebenen Person, oder hat die Frau des Alkoholikers doch recht, wenn sie uns klagt, nicht mehr denselben Mann zu Hause zu haben, den sie einst geheiratet hat? Bewirken unsere medizinischpharmakologischen Bemühungen nur eine Korrektur von defekten Eigenschaften, oder verändern wir damit gleichzeitig auch den Patienten in seiner inneren Einheit? Nicht nur die Metaphysiker unter den Philosophen, sondern auch wir Ärzte und Ärztinnen würden, dem spontanen Empfinden entsprechend, den ersten Teil dieser Fragen mit ja und den zweiten wohl eher mit nein beantworten. David Hume wäre da wahrscheinlich anderer Meinung gewesen, und diese Vermutung hat, auch über zweihundert Jahre nach seinem Tod,

\section{So untersteht das den Lebenslauf der}

Patienten begleitende Ich konsequenterweise auch keiner besonderen Schutzwürdigkeit.

keineswegs an Bedeutung verloren. Im Gegenteil: Die dem strikten Empirismus eigene Reduktion des Substanzbegriffs, und damit letztlich auch der persönlichen menschlichen Identität, auf ein psychologischfiktives Epiphänomen veränderlicher Umstände und Wahrnehmungen sollte gerade uns Ärzte und Ärztinnen auch heute noch aufhorchen lassen. Wenn es nämlich gar keine substanziellen Persönlichkeiten gibt, so ist das den Lebenslauf unserer Patienten begleitende Ich nicht nur der stetigen Veränderung preisgegeben, sondern untersteht konsequenterweise auch keiner besonderen Schutzwürdigkeit. Dies öffnet einer schrankenlosen wissenschaftlich-experimentellen Medizin Tür und Tor. Diese brisante Schlussfolgerung muss nun nicht dazu führen, uns von der liebgewonnenen empirischen Methodik der Versuchsanordnung und Beobachtung zu verabschieden. Es reicht vollkommen aus, dieselbe von Zeit zu Zeit zu hinterfragen und uns vielleicht an die von Hume aufgezeigten Probleme $\mathrm{zu}$ erinnern, die einem einseitig erfahrungswissenschaftlichen Denken innenwohnen. 